\title{
Speeding up coeliac disease diagnosis in cardiological settings
}

Daniela Chicco ${ }^{1}$, Andrea Taddio², Gianfranc Sinagra ${ }^{1}$, Andrea Di Lenarda ${ }^{1}$, Fortunato Ferrara ${ }^{2}$, Michele Moretti ${ }^{1}$, Stefano Martelossi², Nicola Di Toro², Alessandro Ventura², Tarcisio Not ${ }^{2}$

\begin{abstract}
${ }^{1}$ Cardiovascular Department, Azienda Ospedaliero-Universitaria di Trieste, Trieste, Italy 2Department of Reproductive and Development Science, University of Trieste, Institute of Child Health IRCCS "Burlo Garofolo", Trieste, Italy
\end{abstract}

Submitted: 23 June 2009

Accepted: 27 September 2009

Arch Med Sci 2010; 6, 5: 728-732

DOI: $10.5114 /$ aoms.2010.17088

Copyright $\odot 2010$ Termedia \& Banach

\section{Abstract}

Introduction: High prevalence of coeliac disease (CD) has been reported among patients with idiopathic dilated cardiomyopathy (DCM). We evaluated the feasibility and diagnostic accuracy of screening for CD by rapid test of antitransglutaminase antibodies in the cardiology outpatients' clinic.

Material and methods: We screened the blood samples of 104 patients with DCM, 44 of their first-degree relatives, 63 diseased controls and 101 healthy controls for the presence of anti-transglutaminase antibodies in a drop of whole blood using a rapid assay. This test was compared to the enzyme-linked immunosorbent assay and the anti-endomysium antibody test.

Results: Our rapid test was positive in three $(2.9 \%)$ DCM patients, in one $(2 \%)$ relative and in one (1\%) healthy control. These subjects were positive at both control assays. Two DCM patients had iron-deficient anaemia. The healthy relative was asymptomatic, while the healthy control experienced extreme asthenia. The relative refused intestinal biopsy, while the others showed histological evidence of CD. During the gluten-free diet, the patient with the worst left ventricular ejection fraction (LVEF) underwent heart transplant, and LVEF values improved in the other two. Anaemia and tiredness resolved in all patients.

Conclusion: Early detection of $C D$ in a cardiological setting allows prompt treatment with a gluten-free diet of gluten-dependent complaints with potential benefits for the course of DCM.

Key words: anti-transglutaminase antibodies, coeliac disease, dilative cardiomyopathy, screening.

\section{Introduction}

Coeliac disease (CD) is a genetically determined lifelong intolerance to gluten from dietary cereals, with a $1 \%$ prevalence among the general population, but a much higher one (8-20\%) among at-risk populations (first-degree relatives of coeliacs, and patients with autoimmune disorders) $[1,2]$. In CD, regular ingestion of wheat, rye and barley induces T-cell mediated inflammation in the gut and an autoimmune response to selfproteins, mainly tissue type-2-transglutaminase, with the production of anti-tissue-transglutaminase antibodies (anti-tTG) [3].

In general, the classic clinical presentation is seen in subjects with gastroenterological complaints comprising chronic diarrhoea, abdominal

\author{
Corresponding author: \\ Tarcisio Not, MD \\ Department of Reproductive \\ and Development Science \\ University of Trieste \\ Institute of Child Health \\ IRCCS "Burlo Garofolo" \\ via dell'Istria 65/1 \\ 34100 Trieste, Italy \\ Phone: +39040 3785472 \\ Fax: +39 0403785540 \\ E-mail: not@burlo.trieste.it
}


distention and pain, and sometimes malabsorption. In the now more common atypical presentations of the disease, gastrointestinal symptoms may be absent or less pronounced, and extra-intestinal features may be present such as anaemia, osteoporosis, short stature, infertility, neurological and cardiological problems, and associated autoimmune diseases [2]. Because atypical presentations are increasingly found to predominate, $C D$ is now considered a multi-system disorder involving all medical specialities for its diagnosis. Early diagnosis and treatment with a gluten-free diet (GFD) significantly reduces mortality and prevalence of $\mathrm{CD}$-associated disorders [4]. Although definite diagnosis of CD is still based on certain characteristic histological changes in the jejunal intestine, serological tests for CD screening based on detection of anti-tTG are cheaper and less invasive, with excellent sensitivity (98\%) and specificity (95\%) [5]. Given the high prevalence of the disease, the implications of missing diagnosis (or late recognition), and the involvement of all the medical specialities, simple blood drop based immunoassays have been developed and validated for measuring anti-tTG, in order to speed up CD diagnosis in gastroenterology settings [6] and screen the general population [7].

Although the association between CD and heart diseases is not clearly established [8,9], several reports indicate a higher CD prevalence among patients with idiopathic dilated cardiomyopathy (DCM) and myocarditis [10-12].

In a very recent systematic revision a higher prevalence of CD was confirmed among patients with cardiomyopathy; in this subgroup of patients there was also reported an improvement of cardiac performance after introduction of the GFD. Based on these data the authors suggested to consider routine CD screening in all patients suffering from cardiomyopathy [13].

Here, we explore the feasibility of screening patients with DCM and their first-degree relatives for CD using a rapid anti-tTG assay, to be performed by a nurse or cardiologist in the course of daily practice in a third-level referral clinic for cardiology, and we compared the rapid test results with those obtained by using a well-established immunoenzymatic assay.

\section{Material and methods}

\section{Subjects}

From April 2007 to February 2008, 312 subjects underwent screening: 104 adults with DCM (59 males, 45 females, median age 52 years, range 28-61), 44 first-degree relatives of these patients (27 males, 17 females, median age 44 years, range 18-55), 63 diseased controls (43 males, 20 females, median age 59 years, 39-72) and 101 apparently healthy nurses and residents working in cardiology or paediatric departments (60 males, 41 females, median age 40 years, range 27-50). The diagnosis of DCM was based on WHO criteria at the Cardiovascular Department, Azienda OspedalieraUniversitaria of Trieste [14].

Diagnosis of DCM was based on clinical evaluation, 12-lead ECG, echocardiography (global and regional kinetic evaluation, ventricular size, valvular regurgitations, haemodynamic evaluation), Holter monitoring, exercise test and, when indicated, coronary angiography, invasive haemodynamic evaluation, and endomyocardial biopsy. Relatives who agreed to be screened underwent clinical evaluation, 12-lead ECG and echocardiography (global and regional myocardial function, ventricular size, valvular regurgitations, and haemodynamic evaluation). Diseased controls were suffering from acute coronary syndrome $(n=42)$, acute pericarditis $(n=3)$, valvular diseases $(n=8)$, and severe ischaemic heart failure $(n=10)$.

\section{Laboratory testing for coeliac disease}

The rapid test for detecting IgA anti-tTG, based on a whole blood drop, was used as previously described [6] following the manufacturer's instructions (Eu-tTG Quick, Eurospital, Italy). AntitTG were measured by nurses using sticks incubated for 5 minutes in a solution containing $10 \mu$ l of whole blood from a finger prick. Positive results appear as varying shades of grey bands on the sticks (Figure 1). The shades were interpreted by two operators blind to subjects' histories and laboratory findings.

At the same time, the nurses took an additional $80 \mu$ of finger prick blood which was centrifuged: the supernatant was tested for both IgA and IgG anti-tTG using an ELISA assay (Eu-tTG IgG, human IgA, Eurospital, Italy), following the manufacturer's instructions and for IgA anti-endomysium antibodies (AEA), by using an immunofluorescence assay on human umbilical cord cryosections as previously described [15]. These assays were performed 5-10 days after the rapid test by two operators blind to the rapid test results. Subjects testing negative for anti-tTG antibodies were categorized as not having CD.

The DQA1*05/ DQB1 ${ }^{*} 02$ and DQA1*03/DQB1*0302 alleles, encoding the DQ2 and DQ8 heterodimers respectively, were detected in subjects with positive antibody tests by allele-specific PCR, using Dynal SSP Kits (Dynal).

\section{Small bowel biopsies}

Patients positive for anti-tTG or AEA or for both were advised to undergo small intestine biopsy, 


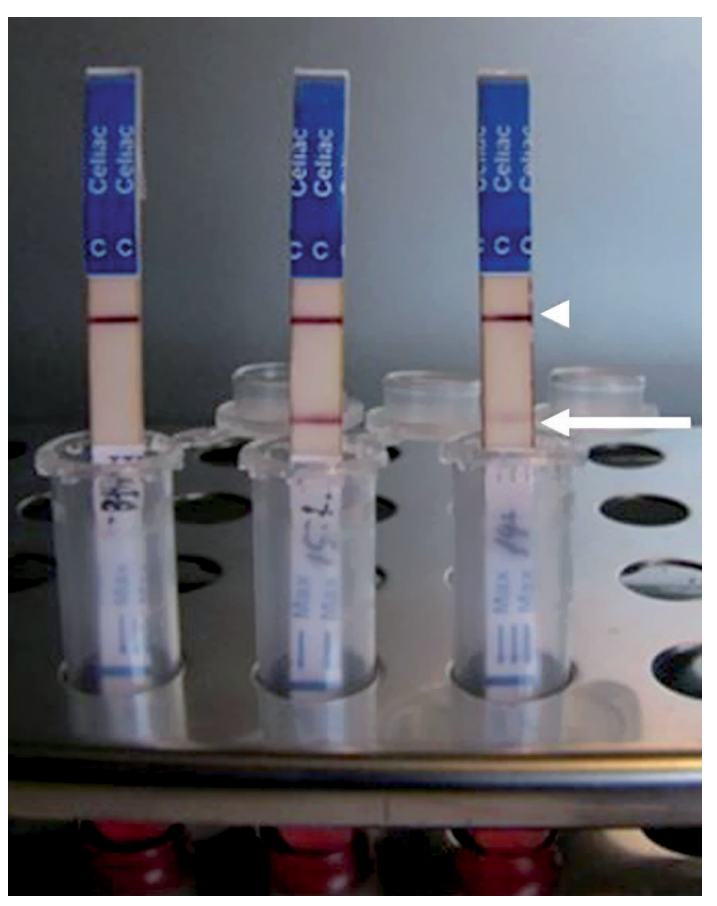

Figure 1. Positive and negative results for antitransglutaminase antibodies using the rapid immunoassay. The first band (head-arrow) must always appear as a technical control, the second band (arrow) represents the positive result of the test. No second band was detected in the first strip incubated with a blood sample that tested negative for anti-transglutaminase antibodies. Different shades of grey bands identified coeliac patients in the second and third strip

analysed according to Oberhuber's classification [16]. Patients whose results were histologically positive were considered to have CD and were started on GFD. The present study was approved by the local independent Ethical Committee.

\section{Results}

Three patients with DCM (2 males, 1 females, 3/104, $2.9 \%), 1 / 44$ (2.2\%) relatives, who was the daughter of a DCM patient, and one nurse among the 101 (1\%) controls tested positive to the rapid test and to both the anti-tTG ELISA and AEA assays. All 5 carried the HLA DQ2 heterodimer: four agreed to undergo intestinal biopsy which showed total villous flattening (type 3c) in all cases. The DCM patient's daughter refused intestinal biopsy, but due to the extremely high positive predictive value (100\%) of co-presence of AEA, anti-tTG and CD-related HLA, this subject was considered as having CD. The remaining 307 subjects (101 DCM patients; 63 sick controls; 100 healthy controls; 43 first-degree relatives) who tested negative to the rapid test were confirmed as negative by both anti-tTG and AEA assays.

We did not find differences in rapid test results compared to those obtained by ELISA assay.
At the time of CD diagnosis one DCM patient presented with severe left ventricular dysfunction (LVEF 20\%), one DCM patient complained of recurrent abdominal pain and iron-deficiency anaemia ( $\mathrm{Hb} 9 \mathrm{~g} / \mathrm{dl}$; mean corpuscular volume $68 \mathrm{fl}$; ferritin serum level $6 \mathrm{ng} / \mathrm{ml}$ ), and one DCM patient presented with iron-deficiency anaemia ( $\mathrm{Hb} 11 \mathrm{~g} / \mathrm{dl}$; mean corpuscular volume $71 \mathrm{fl}$; ferritin serum level $11 \mathrm{ng} / \mathrm{ml}$ ) and severe asthenia (Table I).

Twelve out of 101 patients affected by DCM without CD presented with anaemia (mean value $9.5 \pm 2 \mathrm{~g} / \mathrm{dl}$ ); in these patients normal mean corpuscular volume values (mean value $82 \pm 6 \mathrm{fl}$ ) and serum ferritin concentration (mean value 212 $\pm 37 \mathrm{ng} / \mathrm{ml}$ ) were found. The healthy relative was asymptomatic and refused both the diagnosis of $C D$ and diet therapy. The healthy control experienced severe tiredness without anaemia.

Six months after CD diagnosis, the DCM patient with the worst LVEF value (20\%) underwent heart transplant. Over 16 months of GFD, the LVEF values improved in the other two DCM patients (respectively from $38 \%$ to $47 \%$ and from $46 \%$ to $60 \%)$ and the repetitive ventricular arrhythmias detected in one were no longer present in serial Holter recordings. The GFD improved the anaemia in two patients with DCM and tiredness resolved in the third DCM patient and in the healthy control.

\section{Discussion}

This study shows that an easy, reliable test for detecting IgA anti-tTG antibodies makes quick diagnosis of $C D$ possible in a cardiological setting. This rapid assay is just as accurate as specialized laboratory testing, has extremely high sensitivity and specificity values, takes fifteen minutes to obtain a result and fits in comfortably with the nurse's normal working schedule. Being able to administer such a simple test in the course of routine clinical follow-up examinations helps in early detection of CD and, in the case of positive results, in recognizing $C D$ as the causative agent of otherwise not fully understood clinical symptoms such as tiredness and anaemia. Furthermore, this test could be used in a larger study population in order to establish the real prevalence of $C D$ in patients suffering from heart diseases. In adulthood, complaints of a lifetime of tiredness and anaemia are common and they represent the most frequent symptoms found in delayed CD diagnosis. In a recent Canadian coeliac survey [17], $40 \%$ of adult coeliacs had consulted two or more physicians (including a haematologist) for untreatable anaemia prior to CD diagnosis, and the mean delay in $\mathrm{CD}$ diagnosis after onset of symptoms was 11 years. Interestingly, no patients demonstrated DCM clinical signs among this cohort; but this might be due to the fact that only $50 \%$ of the coeliacs actively participated in the clinical survey. 
Table I. Clinical characteristics of patients with dilated cardiomyopathy and coeliac disease before and after the gluten-free diet

\begin{tabular}{|c|c|c|c|}
\hline & Case 1 Female & Case 2 Male & Case 3 Male \\
\hline Disease presentation & $\begin{array}{l}\text { Recurrent perimyocarditis } \\
\text { (DCM post-myocarditis) }\end{array}$ & $\begin{array}{l}\text { Single episode of } \\
\text { perimyocarditis with HF } \\
\text { (DCM post-myocarditis) }\end{array}$ & $\mathrm{DCM}$ \\
\hline $\begin{array}{l}\text { Family history } \\
\text { of myocardial diseases }\end{array}$ & No & No & No \\
\hline Age at DCM diagnosis & 27 years & 30 years & 36 years \\
\hline Age at $C D$ diagnosis & 37 & 35 & 37 \\
\hline Associated complaints & $\begin{array}{l}\text { Anaemia }(11 \mathrm{~g} \%) \\
\text { severe asthenia }\end{array}$ & $\begin{array}{l}\text { Anaemia (9 g\%), } \\
\text { recurrent abdominal pain }\end{array}$ & Chronic fatigue \\
\hline $\begin{array}{l}C V \text { therapeutic changes } \\
\text { after } C D \text { diagnosis }\end{array}$ & No & No & Added bisoprolol \\
\hline \multicolumn{4}{|c|}{ Clinical and laboratory changes during the gluten-free diet } \\
\hline Follow-up [months] & 16 & 6 & 16 \\
\hline NYHA class & $\mathrm{II}-\mathrm{III} \rightarrow \mathrm{I}$ & $\begin{array}{l}\text { IV } \rightarrow \text { heart } \\
\text { transplantation } 6 \text { months } \\
\text { after CD diagnosis }\end{array}$ & $\mathrm{II} \rightarrow \mathrm{I}$ \\
\hline LVEF [\%] & $38 \rightarrow 47$ & $20 \rightarrow$ transplanted & $46 \rightarrow 60$ \\
\hline LVEDd [cm] & $5.8 \rightarrow 5.6$ & $6.3 \rightarrow$ transplanted & $6.3 \rightarrow 6.1$ \\
\hline $\begin{array}{l}\text { Number of ventricular } \\
\text { extrasystoles }\end{array}$ & $1685 \rightarrow 1083$ & Not available & $7953 \rightarrow 921$ \\
\hline $\begin{array}{l}\text { Repetitive ventricular } \\
\text { events (couples, triplets) }\end{array}$ & No & Not available & Yes $\rightarrow$ No \\
\hline Evolution of symptoms & $\begin{array}{l}\text { Severe asthenia } \rightarrow \\
\quad \text { no symptoms }\end{array}$ & $\begin{array}{l}\text { Recurrent abdominal pain, } \\
\text { chronic diarrhoea } \rightarrow \\
\text { no intestinal symptoms }\end{array}$ & $\begin{array}{c}\text { Chronic fatigue } \rightarrow \\
\text { no symptoms }\end{array}$ \\
\hline Hb values [g/dl] & $11 \rightarrow 13$ & $9 \rightarrow 14$ & $14 \rightarrow 14$ \\
\hline IgA -lgG anti-tTG & $+\rightarrow-$ & $+\rightarrow-$ & $+\rightarrow-$ \\
\hline
\end{tabular}

DCM - dilated cardiomyopathy, CD - coeliac disease, HF- heart failure, CV - cardiovascular, NYHA - New York Heart Association, LVEF - left ventricular ejection fraction (normal value > 50\%), LVEDd - left ventricular end diastolic dimension, Hb-haemoglobin, anti-tTG - anti-tissue transglutaminase antibodies

Also in our own patients, anaemia and chronic fatigue had a negative effect on clinical management, which resolved promptly thanks to GFD, with a great improvement in their quality of life. As previously reported [11, 12], iron-deficiency anaemia could be a useful clinical marker for arousing suspicion of $C D$ in patients with cardiomyopathy, and to use the rapid test described above. Interestingly, those patients without $C D$, but presenting anaemia, did not show laboratory findings indicating irondependent anaemia.

Diagnosis of CD in a DCM patient might also help to treat the cardiac dysfunction, through compliance with GFD, as reported by clinical anecdotal experience [13]. However, further studies are needed to confirm the hypothesis of a positive effect of GFD on the course of DCM. In other autoimmune diseases associated with CD, such as autoimmune hepatitis [18], alopecia [19], gluten-dependent ataxia [20] and dermatitis herpetiformis [21], GFD works as a specific therapeutic agent.

This quick and simple test performed on patients with DCM in a day-clinic setting is both effective and cheap (around $€ 6$ per test vs. $€ 16$ for IgA-lgG ELISA assay), and could reduce delays in CD diagnosis and allow prompt treatment with GFD of gluten-dependent symptoms.

\section{Acknowledgement}

This study was funded by the Institute of Child Health I.R.C.C.S. "Burlo Garofolo" Trieste, by grant number C20/05.

\section{References}

1. Tommasini A, Not T, Kiren V, et al. Mass screening for coeliac disease using anti-human transglutaminase antibody assay. Arch Dis Child 2004; 89: 512-5. 
2. Green P, Cellier C. Celiac disease. N Engl J Med 2007; 357: 1731-43.

3. Koning F. Celiac disease: caught between a rock and hard place. Gastroenterology 2005; 129: 1294-301.

4. Corrao G, Corazza GR, Bagnardi V, et al. Mortality in patients with coeliac disease and their relatives: a cohort study. Lancet 2001; 358: 356-61.

5. Rostom A, Dube C, Cranney A, et al. The diagnostic accuracy of serological tests for celiac disease: a systematic review. Gastroenterology 2005; 128: 838-46.

6. Nemec G, Ventura A, Martelossi S, et al. Looking for celiac disease: diagnostic accuracy of two rapid commercial assays. Am J Gastroenterol 2006; 101: 1597-600.

7. Korponay-Szabó IR, Szabados K, Pusztai J, et al. Population screening for coeliac disease in primary care by district nurses using a rapid antibody test: diagnostic accuracy and feasibility study. BMJ 2007; 335: 1244-7.

8. Vizzardi E, Lanzarotto F, Carabellese N, et al. Lack of association of coeliac disease with idiopathic and ischaemic dilated cardiomyopathies. Scand J Clin Lab Invest 2008; 68: 692-5.

9. Elfström P, Hamsten A, Montgomery SM, Ekbom A, Ludvigsson JF. Cardiomyopathy, pericarditis and myocarditis in a population-based cohort of inpatients with coeliac disease. J Intern Med 2007; 262: 545-54.

10. Curione M, Barbato M, De Biase L, Viola F, Lo Russo L, Cardi E. Prevalence of coeliac disease in idiopathic dilated cardiomyopathy. Lancet 1999; 354: 222-3.

11. Not T, Faleschini E, Tommasini A, et al. Celiac disease in patients with sporadic and inherited cardiomyopathies and in their relatives. Eur Heart J 2003; 24: 1455-61.

12. Frustaci A, Cuoco L, Chimenti C, et al. Celiac disease associated with autoimmune myocarditis. Circulation 2002; 105: 2611-8.

13. Curione $M$, Barbato $M$, Cugini $P$, Amato $S$, Da Ros S, Di Bona S. Association of cardiomyopathy and celiac disease: an almost diffuse but still less know entity. A review. Arch Med Sci 2008; 2: 103-7.

14. Di Lenarda A, Pinamonti B, Mestroni L, et al. How the natural history of dilated cardiomyopathy has changed. A review of the Heart Muscle Disease Registry of Trieste. Ital Heart J Suppl 2004; 5: 253-66.

15. Not T, Citta A, Lucchesi A, Torre G, Martelossi S, Ventura A. Anti-endomysium antibody on human umbilical cord vein tissue: an inexpensive and sensitive diagnostic tool for the screening of coeliac disease. Eur J Pediatr 1997; 156: 616-8.

16. Oberhuber G, Granditsch G, Vogelsang $H$. The histopathology of coeliac disease: time for a standardized report scheme for pathologists. Eur J Gastroenterol Hepatol 1999; 11: 1185-94.

17. Cranney A, Zarkadas M, Graham ID, et al. The Canadian celiac health survey. Dig Dis Sci 2007; 52: 1087-95.

18. Kaukinen K, Halme L, Collin P, et al. Celiac disease in patients with severe liver disease: gluten-free diet may reverse hepatic failure. Gastroenterology 2002; 122: 881-8.

19. Corazza GR, Andreani ML, Venturo N, Bernardi M, Tosti A, Gasbarrini G. Celiac disease and alopecia areata: report of a new association. Gastroenterology 1995; 109: 1333-7.

20. Hadjivassiliou M, Grünewald R, Sharrack B, et al. Gluten ataxia in perspective: epidemiology, genetic susceptibility and clinical characteristics. Brain 2003; 126: 685-91.

21. Collin P, Reunala T. Recognition and management of the cutaneous manifestations of celiac disease: a guide for dermatologists. Am J Clin Dermatol 2003; 4: 13-20. 